\title{
On the Existence of Solutions to Boundary Value Problem of Resonance Fourth- order p-Laplace with One Order Derivative
}

\author{
Fei Yang ${ }^{1, ~ a, ~ Y u a n J i a n ~ L i n ~}{ }^{2, b}$ \\ ${ }^{1}$ Nanchang Institute of Science and Technology, Nanchang 330108, Jiangxi \\ ${ }^{2}$ Nanchang Institute of Science and Technology, Nanchang 330108, Jiangxi \\ afeixu126@126.com, blinyuanzhou@126.com
}

Keywords: resonance, p-Laplace, theory of coincidence degree, boundary value problem

Abstract: This paper deals with the fourth-order p-Laplace boundary value problem of resonance
$\left\{\begin{array}{c}\left(\varphi_{p}\left(x^{\prime \prime}(t)\right)\right)^{\prime \prime}=f\left(t, x(t), x^{\prime}(t)\right), \quad 0<t<1 \\ x(0)=0, x(1)=a x(\xi), x^{\prime \prime}(0)=0, x^{\prime \prime}(1)=b x^{\prime \prime}(\eta)\end{array}\right.$ where $0<\xi, \eta<1 ; a, b>0$ such that $a \xi=1$ and
$b^{p-1} \eta \leq 1$. The existence of solution is obtained by means of Mawhin s continuation theorem.

\section{Introduction}

Boundary value problems of differential equations are of great significance both in theory and in practice. where, differential equation with p-Laplace operator is an important research field in linear analysis, Many practical problems are translated into the existence of solutions to boundary value problems with p-Laplac operatorse . For example, the application of gas dynamics, research on flight stability of missiles, neuroscience and chemistry ${ }^{[1-3]}$. The study of boundary value problems with p-Laplace operator resonance differential equations can not only improve the basic mathematical theory, but also have an important influence on the study of other disciplines ${ }^{[4-5]}$.

$\mathrm{Lu}, \mathrm{Jin}[6]$ proved the existence of solutions for the following boundary value problems is studied by using the coincidence degree theory

$\left\{\begin{array}{c}\left(\varphi_{p}\left(u^{\prime \prime}(t)\right)\right)^{\prime \prime}=f\left(t, u(t), u^{\prime}(t), u^{\prime \prime}(t)\right), 0<t<1 \\ u(0)=0, u(1)=a u(\xi), u^{\prime \prime}(0)=0, u^{\prime \prime}(1)=b u^{\prime \prime}(\eta)\end{array}\right.$

For this boundary value problem, if $a=b=0$ and $f(t, u)$ is nonlinear term, By the fixed point theory proved the existence and multiplicity of some solutions [7-8]. In [9], by using the upper and lower solution method proved the existence result of the solution. These studies on boundary value problems are conducted in non-resonant situations. Based on the above results, In this paper, the existence of solutions to the following boundary value problems is studied by using the coincidence degree extension theorem

$$
\left\{\begin{array}{cc}
\left(\varphi_{p}\left(x^{\prime \prime}(t)\right)\right)^{\prime \prime}=f\left(t, x(t), x^{\prime}(t)\right), & 0<t<1 \\
x(0)=0, x(1)=a x(\xi), x^{\prime \prime}(0)=0, x^{\prime \prime}(1)=b x^{\prime \prime}(\eta)
\end{array}\right.
$$

where $\varphi_{p}(t)=|t|^{p-2} t, f: C\left([0,1] \times R^{2} \rightarrow R\right), 0<\xi, \eta<1, a, b>0$, and $a \xi=b^{p-1} \eta=1$.

\section{Mawhin's continuation theorem:}

Let $X, Y$ be the Banach space, $L: \operatorname{dom} L \subset X \rightarrow Y$ be the Linear mapping, $N: X \rightarrow Y$ be the Nonlinear continuous mapping, Let $\operatorname{dim} \operatorname{ker} L=\operatorname{dim}(Y / \operatorname{Im} L)<+\infty$, and $\operatorname{Im} L$ is a Closed set in $Y$, according to $L$ is a Fredholm operator whose index is zero. If $L$ is a Fredholm operator whose index is zero, then there is a continuous projection operator $P: X \rightarrow \operatorname{Ker} L$ and $Q: Y \rightarrow Y_{1}$, such that $\operatorname{Im} P=K \operatorname{er} L, K \operatorname{er} Q=\operatorname{Im} L, X=\operatorname{Ker} L \oplus \operatorname{Ker} P, Y=\operatorname{Im} L \oplus \operatorname{Im} Q . L_{P}:=\left.L\right|_{\text {domL } \cap X_{1}}$ is invertible, so let's call that the inverse $K$.If $Q N(\bar{\Omega})$ is bounded, and $K(I-Q) N: \bar{\Omega} \rightarrow X$ is relatively tight in $X$, according to $N$ is $L$ - tight in $\bar{\Omega}$, where $\Omega$ is any bounded open set in $X$. 
Lemma 1.1: (Mawhin coincidence degree theory ${ }^{[10]}$ ) Let $X, Y$ be the Banach space, $\mathrm{L}$ is a Fredholm operator whose index is zero, $N: \bar{\Omega} \rightarrow Y$ is $L-$ tight in $\bar{\Omega}$.If

(1) $L x \neq \lambda N x, \forall(x, \lambda) \in($ domL $\cap \partial \Omega) \times(0,1)$;

(2) $N x \in \operatorname{Im} L, \forall x \in \operatorname{Ker} L \cap \partial \Omega$;

(3) $\operatorname{deg}(J Q N, \Omega \cap \operatorname{KerL}, 0) \neq 0$, there $J: \operatorname{Im} Q \rightarrow \operatorname{KerL}$ is a linear isomorphism; equation $L x=N x$ has at least one solution in $\operatorname{dom} L \cap \bar{\Omega}$.

Lemma 1.2 ${ }^{[11]}$ : Let $0 \leq c<\frac{1}{\xi}$, if $v \in[0,1]$, BVP $\left\{\begin{array}{l}x^{\prime \prime}(t)=v(t), t \in(0,1) \\ x(0)=0, x(1)=a x(\xi)\end{array}\right.$ has a unique solution $x$, $x(t)=\int_{0}^{1} \Gamma(t, s) v(s) d s, t \in[0,1]$

$$
\Gamma(t, s)=\left\{\begin{array}{l}
s \in[0, \xi]: \begin{cases}\frac{t}{1-c \xi}[(1-s)-c(\xi-s)], t \leq s \\
\frac{s}{1-c \xi}[(1-t)-c(\xi-t)], s \leq t\end{cases} \\
s \in[\xi, 1]: \begin{cases}\frac{1}{1-c \xi} t(1-s), & t \leq s \\
\frac{1}{1-c \xi}[s(1-t)+t(t-s)], s \leq t\end{cases}
\end{array}\right.
$$

Define 1.1: Let $W=\left\{x: x, \varphi_{p}\left(x^{\prime \prime}\right) \in C^{2}[0,1]\right\}$, if $x \in W$ and satisfies (1), according to $x$ is a solution to the boundary value problem (1).

2. $a \xi=b \eta^{p-1}=1$

When $p \neq 2,\left(\varphi_{p}\left(x^{\prime \prime}\right)\right)^{\prime \prime}=\left(\left|x^{\prime \prime}\right|^{p-2} x^{\prime \prime}\right)^{\prime \prime}$ is nonlinear, so apply the Mawhin's coincidence degree theory, we have BVP (1) in the following form:

$$
\left\{\begin{array}{c}
u_{1}^{\prime \prime}(t)=\varphi_{q}\left(u_{2}(t)\right)=\left|u_{2}(t)\right|^{q-2} u_{2}(t) \\
u_{2}^{\prime \prime}(t)=f\left(t, u_{1}(t), u_{1}^{\prime}(t)\right) \\
u_{1}(0)=0, \quad u_{1}(1)=a u_{1}(\xi) \\
u_{2}(0)=0, \quad u_{2}(1)=b^{p-1} u_{2}(\eta)
\end{array}\right.
$$

There $q>1$ is a constant, and $\frac{1}{p}+\frac{1}{q}=1$. If $u(t)=\left(u_{1}(t), u_{2}(t)\right)^{T}$ is a solution to $(2)$, then $u_{1}(t)$ is a solution to BVP (1).

Define $|\phi|_{0}=\max _{t \in[0,1]}|\phi(t)|, U=\left\{u=\left(u_{1}(\cdot), u_{2}(\cdot)\right)^{T} \in C^{1}[0,1] \times C^{1}[0,1]\right\}$, with the norm $\|u\|_{U}=\max \left\{\left|u_{1}\right|_{0},\left|u_{1}^{\prime}\right|_{0},\left|u_{2}\right|_{0},\left|u_{2}^{\prime}\right|_{0}\right\} ; V=\left\{v=\left(v_{1}(\cdot), v_{2}(\cdot)\right)^{T} \in C^{1}[0,1] \times C^{1}[0,1]\right\}$, the norm $\|v\|_{V}=\max \left\{\left|v_{1}\right|_{0},\left|v_{2}\right|_{0}\right\} . U$ and $V$ be the Banach space. Let $L: \operatorname{dom} L \subset U \rightarrow V$, and

$$
L u=\left(\begin{array}{l}
u_{1}^{\prime \prime} \\
u_{2}^{\prime \prime}
\end{array}\right)
$$

where $\operatorname{dom} L=\left\{u \in C^{1}[0,1] \times C^{1}[0,1]: u_{1}(0)=0, u_{2}(1)=a u_{1}(\xi), u_{2}(0)=0, u_{2}(1)=b^{p-1} u_{2}(\eta)\right\}$ Let $N: U \rightarrow V$, and 


$$
N u=\left(\begin{array}{c}
\varphi_{q}\left(u_{2}(t)\right) \\
f\left(t, u_{1}(t), u_{1}^{\prime}(t)\right)
\end{array}\right)
$$

so (2) is transformed into an abstract equation: $L u=N u, u \in d o m L$.

It's easy to see by the definition of $L, \operatorname{Ker} L=\left\{u=\left(c_{1} t, c_{2} t\right)^{T}: c_{1}, c_{2} \in R\right\}$ and $\operatorname{Im} L=\left\{v=\left(v_{1}(\cdot), v_{2}(\cdot)\right)^{T} \in V: \int_{0}^{1} \int_{\xi t}^{t} v_{1}(s) d s d t=\int_{0}^{1} \int_{\eta t}^{t} v_{2}(s) d s d t=0\right\}$, Assumed projection operator $P: U \rightarrow \operatorname{Ker} L$ and $Q: Y \rightarrow \operatorname{Im} Q$ as follows $(P u)(t)=\left(u_{1}^{\prime}(0) t, u_{2}^{\prime}(0) t\right)^{T}$, $(Q v)(t)=\left(\frac{2}{1-\xi} \int_{0}^{1} \int_{\xi t}^{t} v_{1}(s) d s d t, \frac{2}{1-\eta} \int_{0}^{1} \int_{\xi t}^{t} v_{1}(s) d s d t\right)^{T}$

For $v \in V$, let $\tilde{v}=v-Q v$, so $\int_{0}^{1} \int_{\xi t}^{t} \tilde{v}_{1}(s) d s d t=\int_{0}^{1} \int_{\eta t}^{t} \tilde{v}_{2}(s) d s d t=0 . \tilde{v} \in \operatorname{Im} L$ and $V=\operatorname{Im} L+R^{2}$. $\operatorname{In}$ addition, $\operatorname{Im} L \cap R^{2}=\{0\}$, thus $V=\operatorname{Im} L \oplus R^{2}$.That means $\operatorname{dim} \operatorname{Ker} L=\operatorname{codim} \operatorname{Im} L<+\infty$, so $L$ is an Fredholm operator whose index is zero. On the other hand, $K$ is $\left.L\right|_{\text {KerP^domL }}$ inverse, so $(K v)(t)=\left(\int_{0}^{t} \int_{0}^{\tau} v_{1}(s) d s d \tau, \int_{0}^{t} \int_{0}^{\tau} v_{2}(s) d s d \tau\right)^{T}$

By (4) and (5), we have $N$ is $L$ - tight in $\bar{\Omega}, \Omega$ is any bounded open set in $U$.

Theorem 2.1: If $a \xi=b^{p-1} \eta=1$, and satisfying

$\left(\mathrm{H}_{1}\right)$ there is constant $D>0$ such that $v f(t, u, v)>0$ (or $\left.v f(t, u, v)<0\right)$, for all $|v|>D, t \in[0,1]$ and $u \in R$.

$\left(\mathrm{H}_{2}\right)$ there is a nonnegative constant $r_{i}, i=1,2,3,4,5$ such that

$$
|f(t, u, v)| \leq r_{1}|u|^{p-1}+r_{2}|v|^{p-1}+r_{3},(t, u, v) \in[0,1] \times R^{2}
$$

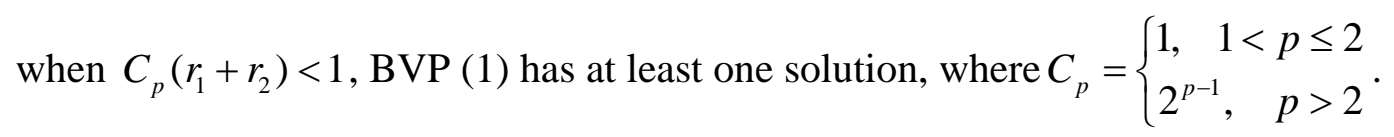

Proof: For the equation $L u=\lambda N u, \lambda \in(0,1)$. Let

$\Omega_{1}=\{u \in \operatorname{dom} L: L u=\lambda N u, \lambda \in(0,1)\}$, If $u(t)=\left(u_{1}(t), u_{2}(t)\right)^{T} \in \Omega_{1}$, then

$$
\left\{\begin{array}{c}
u_{1}^{\prime \prime}(t)=\varphi_{q}\left(u_{2}(t)\right)=\left|u_{2}(t)\right|^{q-2} u_{2}(t) \\
u_{2}^{\prime \prime}(t)=f\left(t, u_{1}(t), u_{1}^{\prime}(t)\right) \\
u_{1}(0)=0, \quad u_{1}(1)=a u_{1}(\xi) \\
u_{2}(0)=0, \quad u_{2}(1)=b^{p-1} u_{2}(\eta)
\end{array}\right.
$$

First we prove that there is a constant $t_{1}, t_{2} \in[0,1]$, such that

$$
\begin{gathered}
\left|u_{1}^{\prime}\left(t_{1}\right)\right| \leq D \\
u_{2}\left(t_{2}\right)=0
\end{gathered}
$$

In fact, by $L u=\lambda N u$, we get $Q N x=0$, thus $\left.\int_{0}^{1} \int_{\eta t}^{t} f\left(s, u_{1}(s), u_{1}^{\prime}(s)\right)\right) d s d t=0$, so, there are $t_{1} \in[0,1]$, such that $f\left(t_{1}, u_{1}\left(t_{1}\right), u_{1}^{\prime}\left(t_{1}\right)\right)=0$, by $\left(\mathrm{H}_{1}\right)$, we get (7) set up.

On the other hand, by boundary conditions and functions $u_{1}(t)$ is continuous in $[0,1]$, we get $\xi_{1} \in(0, \xi)$ 和 $\xi_{2} \in(\xi, 1)$, such that $a u_{1}(\xi)-u_{1}(\xi)=(a-1)\left[u_{1}(\xi)-u_{1}(0)\right]=(1-\xi) u_{1}^{\prime}\left(\xi_{1}\right)$, $u_{1}(1)-u_{1}(\xi)=(1-\xi) u_{1}^{\prime}\left(\xi_{2}\right)$; there are $u_{1}^{\prime}\left(\xi_{1}\right)=u_{1}^{\prime}\left(\xi_{2}\right), \xi_{3} \in\left(\xi_{1}, \xi_{2}\right) \subset(0,1)$, such that $u_{1}^{\prime \prime}\left(\xi_{3}\right)=0$, then $u_{2}\left(\xi_{3}\right)=\varphi_{p}\left(u_{1}^{\prime \prime}\left(\xi_{3}\right)\right)=0$.by $u_{2}(0)=0$ and $u_{2}(t)$ is continuous in $[0,1]$, we gen (8) set up. 
The second, by (6) and $\left(\mathrm{H}_{2}\right)$, we get

$$
\begin{aligned}
\int_{0}^{1}\left|u_{2}^{\prime \prime}(t)\right| d t & =\lambda \int_{0}^{1}\left|f\left(t, u_{1}(t), u_{1}^{\prime}(t)\right)\right| d t \\
\leq & r_{1} \int_{0}^{1}\left|u_{1}(t)\right|^{p-1} d t+r_{2} \int_{0}^{1}\left|u_{1}^{\prime}(t)\right|^{p-1} d t+r_{3} \\
\leq & r_{1}\left|u_{1}\right|_{0}^{p-1}+r_{2}\left|u_{1}^{\prime}\right|_{0}^{p-1}+r_{3}
\end{aligned}
$$

By (7)(8) and Hölder inequality, we have

$$
\begin{aligned}
& \left|u_{1}\right|_{0} \leq\left|\int_{0}^{t} u_{1}^{\prime}(s) d s\right| \leq\left|u_{1}^{\prime}\right|_{0} \leq\left|u_{1}^{\prime}\left(t_{1}\right)+\int_{t_{1}}^{t} u_{1}^{\prime \prime}(s) d s\right| \leq D+\int_{0}^{1}\left|u_{1}^{\prime \prime}(s) d s\right| \\
& \left|u_{2}\right|_{0} \leq\left|\int_{0}^{t} u_{2}^{\prime}(s) d s\right| \leq\left|u_{2}^{\prime}\right|_{0} \leq\left|u_{2}^{\prime}\left(t_{2}\right)+\int_{t_{2}}^{t} u_{2}^{\prime \prime}(s) d s\right| \leq \int_{0}^{1}\left|u_{2}^{\prime \prime}(s)\right| d s
\end{aligned}
$$

By (6), we get $\int_{0}^{1}\left|u_{1}^{\prime \prime}(s)\right| d s=\lambda \int_{0}^{1}\left|\varphi_{q}\left(u_{2}(t)\right)\right| d s \leq \varphi_{q}\left(\left|u_{2}\right|_{0}\right)$

Substitute equation (10-12) into equation (9), we get

$$
\begin{aligned}
\int_{0}^{1}\left|u_{2}^{\prime \prime}(t)\right| d t & \leq\left(r_{1}+r_{2}\right)\left(D+\varphi_{q}\left(\left|u_{2}\right|_{0}\right)\right)^{p-1}+r_{3} \\
& \leq C_{p}\left(r_{1}+r_{2}\right)\left(D^{p-1}+\left|u_{2}\right|_{0}\right)+r_{3} \\
& \leq C_{p}\left(r_{1}+r_{2}\right) \int_{0}^{1}\left|u_{2}^{\prime \prime}(t)\right| d t+r_{3}
\end{aligned}
$$

by $p>1$ and $C_{p}\left(r_{1}+r_{2}\right)+r_{3}+r_{4}<1$ set up, The above formula indicates that there is a constant $M_{1}>0$, such that $\int_{0}^{1}\left|u_{2}^{\prime \prime}(t)\right| d t \leq M_{1}$

so, $\left|u_{2}\right|_{0} \leq\left|u_{2}^{\prime}\right|_{0} \leq M_{1}$

$$
\left|u_{1}\right|_{0} \leq\left|u_{1}^{\prime}\right|_{0} \leq D+M_{1}^{q-1}:=M_{2}
$$

Let $\Omega=\left\{u \in U:\|u\|_{U}<\max \left\{M_{1}, M_{2}\right\}+1\right\}$, The lemma 1.1 condition (1) is satisfied. Without loss of generality, Assuming that $|v|>D, t \in[0,1]$ and $u \in R, v f(t, u, v)>0$ is set up. So let's prove that for $u \in \operatorname{Ker} L \cap \partial \Omega$ 有 $N u \notin \operatorname{Im} L$. Otherwise, there are $u_{0}=\left(c_{1} t, c_{2} t\right) \in \operatorname{Ker} L$ such that $N u_{0}=\left(\varphi_{q}\left(u_{2}\right), f\left(t, c_{1} t, c_{1}\right) \in \operatorname{Im} L\right.$.That is $Q N u_{0}=0$, so $\int_{0}^{1} \int_{\eta t}^{t} f\left(s, c_{1} s, c_{1}\right) d s d t=0$.According to the condition $\left(\mathrm{H}_{1}\right)$, we get $\left|c_{1}\right| \leq D<M_{2}$, That contradicts $u_{0} \in \partial \Omega$. Therefore, condition (2) in lemma 1.1 is also satisfied.

Let the mapping $J: \operatorname{Im} Q \rightarrow \operatorname{Ker} L$ is $J\left(c_{1}, c_{2}\right)=\left(c_{1} t, c_{2} t\right)$, and $H(u, \mu)=\mu u+(1-\mu) J Q N, \forall(u, \mu) \in \bar{\Omega} \times[0,1]$; For $u \in(\partial \Omega \cap \operatorname{KerL}) \times[0,1]$, we have

$$
H(u, \mu)=\left(\begin{array}{c}
\mu c_{1} t+\frac{2(1-\mu)}{1-\eta} \int_{0}^{1} \int_{\eta t}^{t} f\left(s, c_{1} s, c_{1}\right) d s d t \\
\mu c_{2} t+\frac{2(1-\mu)}{1-\xi} \int_{0}^{1} \int_{\xi t}^{t} \varphi_{q}\left(c_{2} s\right) d s d t
\end{array}\right) \neq 0
$$

So, $\operatorname{deg}\{J Q N, \Omega \cap \operatorname{KerL}, 0\}=\operatorname{deg}\{H(0, u), \Omega \cap \operatorname{KerL}, 0\}$

$$
=\operatorname{deg}\{H(1, u), \Omega \cap \operatorname{Ker} L, 0\}=\operatorname{deg}\{I, \Omega \cap \operatorname{Ker} L, 0\} \neq 0,
$$

That is, condition (3) in lemma 1.1 is satisfied. According to lemma 1.1, there is a solution to equation (6) in $\bar{\Omega} \cap \operatorname{domL} u^{*}(t)=\left(u_{1}^{*}(t), u_{2}^{*}(t)\right)^{T}$.so BVP (1) has a solution $u_{1}^{*}(t)$. 


\section{Conclusion}

In this paper, the existence of at least one solutions to boundary value problem of resonance fourth-order p-Laplace with one order derivative is considered; By means of Mawhin's continuation theorem, the existence of solution is verified .

\section{Acknowledgements}

This work is supported by Department of education science and technology research youth project in 2017(Item no.GJJ171107).

\section{References}

[1] Bernis F. Compactness of the support in convex and non-convex fourth order elasticity problems. Nonlinear Anal,1982, 6:1221-1243.

[2] Agarwal P R, Lü H S, O'Regan D. Positive solutions for the boundary value problem $\left(\left|u^{\prime \prime}\right|^{p-2} u^{\prime \prime}\right)^{\prime \prime}-\lambda q(t) f(u(t))=0$. Mem Differential Equations Math Phys,2003,28:33-44.

[3] Jiang D Q,Liu H Z, Xu X J. Nonuerinant singular fourth-order boundary value problems. Appl Math Lett, 2005,18:69-75.

[4] Shen chunfang, Yang liu. Existence of solution for boundary value problem with p-Laplace at resonance. Applied mathematics, 2007, 20(4):783-790.

[5] Jing weihua, Yang caixia. The existence of positive solutions to the problem of resonance boundary value with integral boundary conditions. Journal of Hebei University of Science and Technology,2015,36(4):376-381.

[6] Jin shan, Lu shiping. Existehnce of Solutions for Fourth Order Boundary Value Problem with pLaplace at Resonance. Acta Mathematica Scientia, 2011, 31A (3):829-836.

[7] Bai Z B. Existence and multiplicity of positive solutions for a fourth-order p-Laplace equations. Appl Math Mech, 2001, 22:1476-1480.

[8] Jin Shan, Zhang Zhirong, Lu Shiping. Existence of solutions for a fourth-order p-Laplace equations boundary value problem. Journal of University (science edition) (in Chinese), 2008, 46(2):214-218.

[9] Bai Z B, Ge W G. The iterative solutions for some fourth-order p-Laplace equation boundary value problem. Appl Math Let, 2006,19:8-14.

[10] Gaines R E, Mawhin J L. Coincidence Degree and Nonlinear Differential Equations. Berlin: Springer, 1977.

[11] Guo Y, Ge W G. Positive solutions for three-point boundary value problems with dependence on the first order derivative. J Math Anal Appl, 2004,290:291-301. 